\begin{tabular}{|c|c|c|c|c|c|c|}
\hline \multicolumn{3}{|c|}{$\begin{array}{l}\text { Interval before or } \\
\text { after minimum }\end{array}$} & Light & \multicolumn{2}{|c|}{$\begin{array}{l}\text { Interval before or } \\
\text { after minimum }\end{array}$} & Light \\
\hline \multicolumn{3}{|c|}{ o hours } & 2.0 & I 4 hours & $\ldots$ & 8. $x$ \\
\hline 2 & $»$ & & 2.0 & I 6 & $\ldots \ldots \ldots$ & 8.2 \\
\hline 4 & 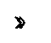 & & 2.1 & I 8 & $\ldots \ldots \ldots$ & 8.3 \\
\hline 6 & $\star$ & & 2 & 20 & $\ldots \ldots$ & 8.3 \\
\hline 8 & » & & 5.0 & 22 & $\ldots \ldots \ldots$ & 8.3 \\
\hline Io & $\gg$ & & 7.1 & 24 & and over & 8.3 \\
\hline I 2 & $\gg$ & . & 7.8 & & & \\
\hline
\end{tabular}

While, of course, the observations are not numerous enough to fix the details of the light variations with great precision, it is manifest that the decrease in light sensibly begins about 12 hours before minimum, occupying 8 hours; the star remains about 4 hours at minimum and then increases in 8 hours again to its normal brightness. It should be stated that it was not until the last observed minimum, or Nov. 25, that any suspicion was entertained as to the peculiar character of the light changes, or as to the true period so that the observations were entirely free from prejudice. My thanks are due to Mr. S. C. Chandler jr. of the Harvard College Observatory for advice as to some of the details of the reduction of the observations.

E phemeris.

Paris M. T. of Minima.

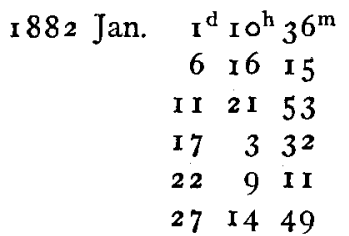

Febr. 12028

726

I 2745

$$
\begin{aligned}
& \text { I } 882 \text { Febr. I } 7^{\mathrm{d}} 13^{\mathrm{h}} 23^{\mathrm{m}} \\
& \begin{array}{lll}
22 & 19 & 2
\end{array} \\
& 28 \circ 40 \\
& \text { Mar. } 56 \text { I9 } \\
& \text { I0 II } 57 \\
& \begin{array}{lll}
5 & \text { I } 7 & 37
\end{array} \\
& 20 \quad 23 \quad 15 \\
& 26 \quad 4 \quad 54 \\
& 3 \text { I } 1034
\end{aligned}
$$

Cambridgeport, Mass., U. S. A. 188 I Dec. I 5.

\title{
Beobachtungen über die Helligkeit und den Schweif der Cometen 1881 III und IV.
}

Am 27 . Juni bemerkte ich den Cometen III am Nordhimmel und schätzte die Helligkeit des Kopfes zwischen Wolken $=\mathrm{I}^{\mathrm{m}}$, ebenso am 28 , die Schätzungen sind aber wahrscheinlich zu hell. Der Kopf und einige Grade vom Schweif waren an den folgenden Tagen schon während der Dämmerung kenntlich. Bei eingetretener Dunkelheit gewährte der Comet einen prächtigen Anblick, doch kam er dem $187_{4}^{\text {er }}$, dessen ich mich noch deutlich entsinne, wohl nicht gleich.

Die Helligkeitsvergleiche mit benachbarten Sternen ergeben folgende Daten: Polaris $=2^{\mathrm{m}}{ }_{15} ; \varepsilon$ Cassiopeiae $=3 \cdot 7 ; 5$ Ursae min. $=4 \cdot 7$ angenommen.

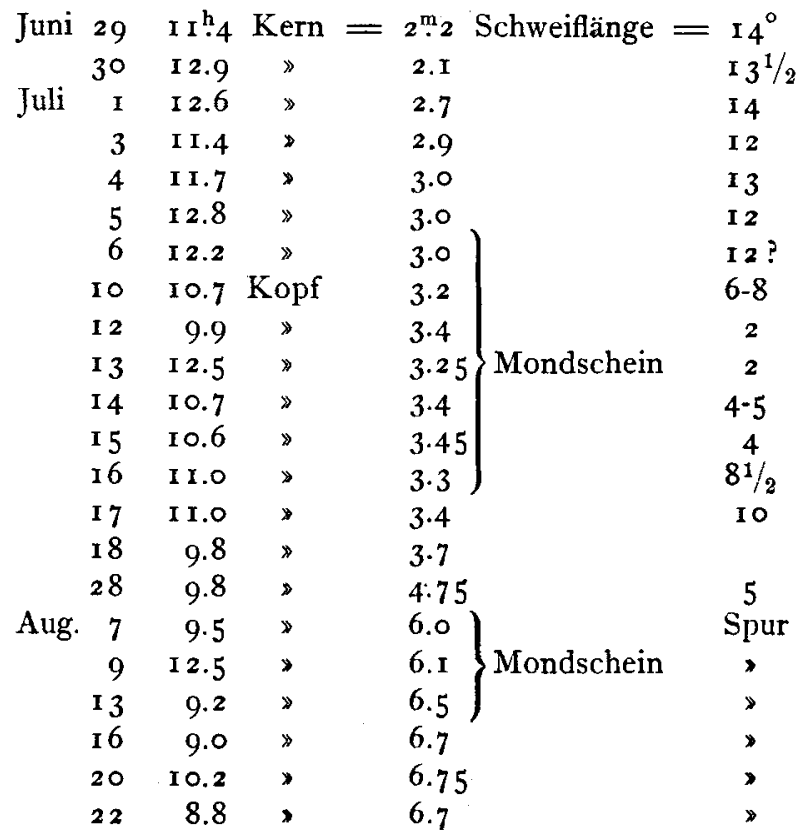

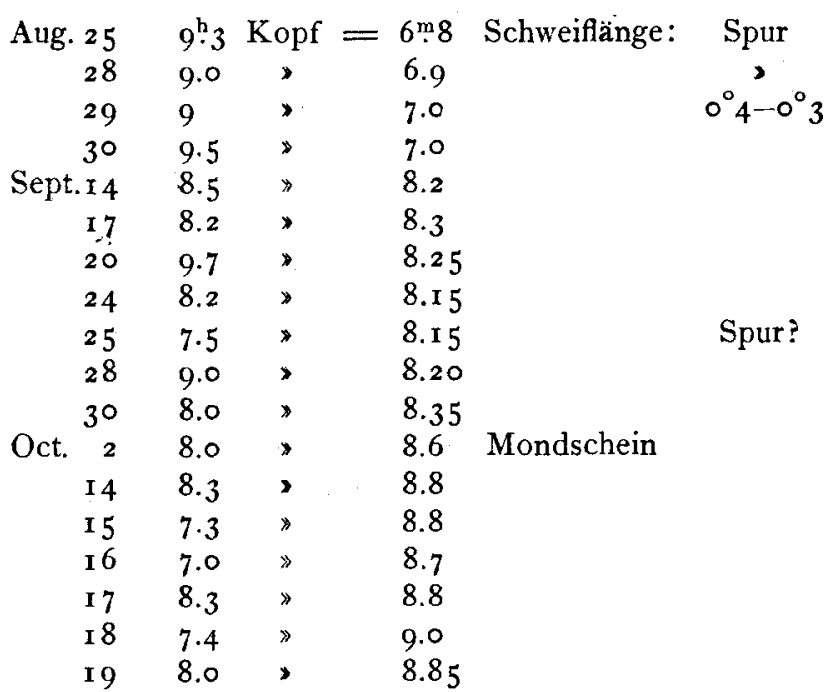

Nach diesen Werthen wurde die Curve der Helligkeitsänderung gezeichnet. Dieselbe ist keineswegs gleichmässig abnehmend, sondern zeigt wiederkehrende Abweichungen, indem etwa von Juli 5-17, dann von Aug. 1 7-26 und gegen Ende des September eine Verzögerung der Abnahme resp. gegen Juli 20, Aug. 30 und Anfang October (unsicher) ein Maximum des Lichtes angezeigt ist. Wegen der den Kern umgebenden Hülle haben die Vergleiche die bei veränderlichen Sternen erlangte Sicherheit natürlich nicht.

Der Stern $56^{\mathrm{m}}$ in $\left.\alpha=89^{\circ}, \delta=+69^{\circ}(1855)^{*}\right)$ stand am 29. Juni mitten im Schweife, in etwa $3-4^{\circ}$ Distanz vom Kern. Vergleiche mit einigen Nachbarn ergaben keine merkliche Veränderung der Helligkeit des Sternes.

Zur Bestimmung der Schweiflänge sind stets nur die
$K r$. 
mit Sicherheit kenntlichen Endtheile auf der Karte verzeichnet worden. Die Helligkeitsabnahme des Schweifs betraf die, in der ersten Zeit fächerförmig ausgebreiteten, Endtheile und besonders den in der Cometenbahn vorangehenden Rand. Im Allgemeinen war der nachfolgende Rand stets schärfer begrenzt als der andere.

Am 29. Juni war ein Strahl oder Theilzweig in dem Cometen näher dem vorausgehenden Rande bemerkbar, der am 30. schon schwächer geworden, am I. Juli mit dem Endtheile sich mehr dem rechten (in der Bahn nachfolgenden) Rande genähert hatte und Juli 3 verschwunden war.

Von Mitte Juli ab ward der Schweif merklich schmäler, gegen Ende des Monats erschien der Comet schon im Ganzen sehr lichtschwach.

Der Kern nahm seit Anfang Juli stetig an Helligkeit ab, die Hülle dagegen in den ersten Tagen eher noch zu. Ausströmungen vom Kern waren zwischen Juli 3 und 6 bemerkbar. Mit blossem Auge war der Kern bis zum 1 5. Juli von der Hülle zu trennen, seit dem 16 . verschwand er in der Helligkeit des Kopfes.

Im Fernrohr (Astr, Nachr. Nr. 2248 mit $B$ bez.), das ich erst seit Juli ro benutzen konnte, erschien der Kern stets viel heller als der Kopf, mit merklichem Durchmesser, planetarischem Lichte und verwaschenem Rande. Am 28. Juli war der Kern noch als solcher kenntlich, am 7. August dagegen schon sehr verschwommen.

Nach dem Mondschein (am 18. Aug.) blieb der Comet für das blosse Auge noch deutlich bis zum 29. Aug. sichtbar, aber nicht so scharf wie Sterne gleicher Helligkeit. Von jetzt $a b$ sah er im Fernrohr einem grossen, hellen, in der Schweifrichtung länglichen Nebel ähnlich, dessen Helligkeit von der Mitte aus bedeutend abnahm, was selbst bei den letzten Beobachtungen trotz grosser Lichtschwäche noch hervortrat.

Eine Periode anhaltender Trübung vereitelte weitere Beobachtungen nach October I9.
Die Erscheinung des Cometen IV war bedeutend lichtschwächer, auch die Figur nicht so scharf begrenzt, als die seines Vorgängers. Nah am Kopfe erschien der Schweif ziemlich hell und von einiger Breite (etwa $1 / 3^{\circ}$ bis $1 / 2^{\circ}$ ), nach dem Ende spitz verlaufend. Die in der Bahn vorangehende Seite war schärfer begrenzt, als die andere, welche kurze, seitliche Ausströmungen vermuthen liess.

Schweiflänge am 1 8. u. 20. $=4^{1} /_{2}^{\circ} ; 22=6^{\circ} ; 25=7^{\circ}$; 30. etwa $4^{\circ}$. Am 29. u. 30. erschien der Schweif so schwach, dass man, besonders am letzten Tage, den Cometen bei füchtigef Beobachtung leicht für einen Fixstern halten konnte, wobei wohl die ungünstige Stellung am westl. Horizont mitwirkte.

Im Fernrohr $(B)$ zeigte sich der Kern bedeutend heller als die umgebende Hülle, mit deutlichem Durchmesser und planetarischem Lichte.

Aus den Vergleichen des Cometen mit einigen Nachbarsternen ergaben sich für die Lichtstärke des Kopfes folgende Grössenangaben.

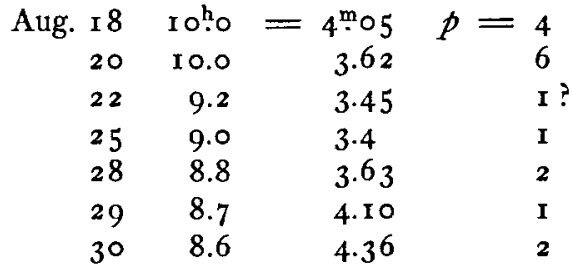

Mit diesen Werthen wurde die Zeit des Maximums der Helligkeit, unter Berücksichtigung des Gewichtes ( $p$ ) durch eine Curvenzeichnung auf

$$
\text { August } 24.2 \text { Lichtstärke }=3^{\mathrm{m}} \cdot 4
$$

bestimmt. Die $4^{\mathrm{m}}$.0 erreichte der Comet August 18.6 und 29.3.

Angenommene Sternhelligkeiten: Cor Caroli $=3^{\mathrm{m}} \mathbf{2}$; $\delta$ Ursae majoris $=3^{\mathrm{m}} \cdot 8 ; \chi$ Urs. maj. $=4^{\mathrm{m}} \cdot 5$.

Frankfurt a/M. I 88I im December.

\section{Friedrich Schwab.}

\section{Entdeckung eines neuen Planeten (221). Von Herrn F.Palisa.}

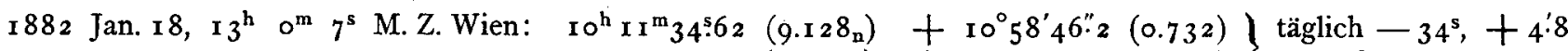

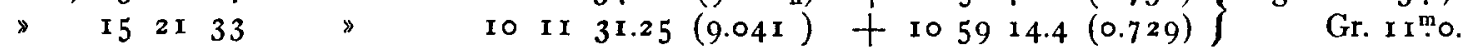

Berichtigungen zu Nr. 2269 (Bd.95) pag. I95 unter Mira Ceti 1878/79 fehlt: Maximum: 1878 October 21.

195 Zeile 13 von unten 1878 März soll sein : 1879 März.

197, II, oben ist Anfang (November) einzuschalten.

- zum Register, Bd, Ioo, 395 1.: Millosevich, E., Prof., Astronom und Vicedirector der Sternwarte des Coll. Romano in Rom.

- 399 1.: Riccò, A., Prof., Erster Astronom an der Sternwarte zu Palermo.

- 404 l.: Zona, T., Zweiter Astronom an der Sternwarte zu Palermo.

\section{In ha $1 \mathrm{t}$ :}

Zu Nr. 241 2. Rob. Luther. Kreismicrometer-Beobachtungen von kleinen Planeten am Sicbenfüsser zu Duisseldorf. I 77. - A. Winnecke. Beobachtungen von kleinen Planeten am Fadenmicrometer des 6zölligen Bahnsuchers. I83. - H. Pomerantzeff. Observations des petites planètes faites à l'Observatoire de Tachkent à l'aide du micromètre filaire du réfracteur de 6 pouces. I 85 , - Edw. F. Sazoyer. A new variable star. (DM. $+\mathrm{I}^{\circ} 3408 ; 1855.0: \mathrm{r}^{\mathrm{h}} \mathrm{g}^{\mathrm{m}} \mathrm{I}^{\mathrm{s}}+\mathrm{I}^{\circ} 22^{\prime} 6$ ). $187 .-$ Friedrich Schwab. Beobachtungen über die Helligkeit und Schweiflänge der Coineten 188 I III und 1V. 189. - F Palisa. Entdeckung eines neuen Planeten (221). 191. - Berichtigungen. I I9. 